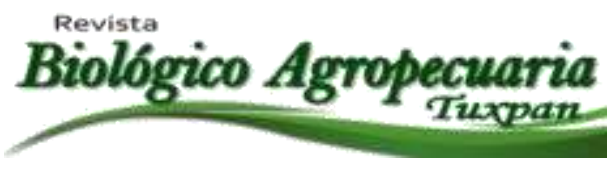

\title{
Indicadores de Estrés en Respuesta a la Inyección Intramuscular de Oxitocina para Inducir la Eyección de Leche en Vacas Lecheras del Trópico Veracruzano
}

Indicators of Stress in Response to Intramuscular Oxytocin Injection to Induce Milk Ejection of Dairy Cows in the Tropics of Veracruz

Lammoglia Miguel Ángel ${ }^{1 凶}$, Estrella Martínez B. ${ }^{1}$, Aedo Braulio ${ }^{1}$, Daniel Iliana C. ${ }^{1}$ y Garcez Nora ${ }^{1}$

${ }^{1}$ Facultad de Ciencias Biológicas y Agropecuarias, Universidad Veracruzana, Poza Rica-Tuxpan Carretera Tuxpan-Tampico, km 7.5, Tuxpan, Ver. 017838344350;

${ }^{\bowtie}$ Autor para correspondencia: malammoglia@ hotmail.com

Recibido: 11/01/2014

Aceptado: 17/07/2014

\section{RESUMEN}

El objetivo fue determinar el efecto de la aplicación intramuscular de oxitocina para inducir la eyección de leche en indicadores de estrés y temperatura rectal en vacas ordeñadas mecánicamente en el trópico veracruzano. Se utilizaron vacas cruzadas $(n=96)$ Suizo Americano-Cebú y Holstein-Cebú ordeñadas 2 veces al día. Las vacas después del parto se asignaron aleatoriamente de acuerdo al numero de partos a recibir 10 UI de oxitocina intramuscularmente $(n=56)$ o NO ser inyectadas $(n=40)$ al ordeñarlas. Durante el ordeño se observaron las siguientes variables: pisoteo, pateo, movimiento de la cola, dejar de comer, si se movió bruscamente y después del ordeño se tomó la temperatura rectal (TR). El análisis estadístico se realizó utilizando ANOVA y ji-cuadrada del software statistica 7. Las vacas inyectadas con oxitocina tuvieron un mayor $(\mathrm{P}=.05)$ porcentaje de animales que pisotearon y patearon $(53 \%)$, dejaron de comer $(61 \%)$, movieron la cola $(71.4 \%)$ y se movieron $(71.5 \%)$ comparadas con las vacas no inyectadas $(10 \%, 0 \%, 3 \%, 3 \%, 6 \%$, respectivamente). Las vacas inyectadas con oxitocina tuvieron una mayor temperatura rectal $\left(39.13 \pm .08{ }^{\circ} \mathrm{C}\right)$ y numero de pisoteos (2.62 \pm .36$)$ comparado con las vacas no inyectadas $\left(38.96 \pm .12{ }^{\circ} \mathrm{C}\right.$ y $.24 \pm .54$; respectivamente). En conclusión, las vacas inyectadas con oxitocina manifestaron elevados indicadores de estrés y temperatura rectal comparados con los de las vacas no inyectadas, sugiriendo que si existe una reacción de las vacas inyectadas intramusculares y rutinariamente con oxitocina y estas no se habitúan a la inyección.

Palabras clave: oxitocina, eyección de leche, indicadores de estrés. 


\section{ABSTRACT}

The objective was to determine the effect of intramuscular injection of oxytocin to induce milk ejection on stress indicators and rectal temperature in cows mechanically milked in the tropics of Veracruz. Crossbred ( $\mathrm{n}=96$ ) cows (American Brown Swiss-Cebu and Holstein-Cebu) milked 2 times a day were used. After calving cows were randomly assigned according to the number of lactations to receive a 10 IU oxytocin intramuscularly injection at milking $(n=56)$ or NOT be injected $(n=30)$. During milking, the following variables were observed: trampling, kicking, tail movement, stop eating, if it moved abruptly and after milking took rectal temperature (RT). Statistical analysis was performed using ANOVA and chi-square using the software Statistica 7. Cows injected with oxytocin had a greater $(\mathrm{P}=$ $.05)$ percentage of animals trampled and kicked (53\%), stopped eating (61\%), moved the tail (71.4\%) and moved abruptly (71.5\%) compared to cows that were Not injected $(10 \%, 0 \%, 3 \%, 3 \%, 6 \%$, respectively). Cows injected with oxytocin had higher rectal temperature $\left(39.13 \pm 0.08{ }^{\circ} \mathrm{C}\right)$ and number of trampling $(2.62 \pm 0.36)$ compared to cows that were Not injected $\left(38.96 \pm 0.12{ }^{\circ} \mathrm{C}\right.$ and $0.24 \pm .54$, respectively). In conclusion, cows injected with oxytocin had increased stress indicators and rectal temperature compared with cows that were not injected. Cows injected intramuscularly and routinely with oxytocin showed a stress reaction; therefore it is possible to suggest that these cows do not habituate to the oxytocin injection.

Keywords: oxytocin, milk ejection, stress indicators.

\section{INTRODUCCIÓN}

En los últimos años se ha incrementado la investigación en el tema de bienestar animal en todas las especies a nivel mundial. Existen un sin número de publicaciones que demuestran que los animales en un estado de bienestar son más productivos y en casos de animales de compañía presentan menos problemas conductuales.

En el trópico veracruzano la mayoría del ganado bovino tiene influencia genética de cebú debido a las condiciones ambientales y esta raza les da una mayor resistencia al ganado Bos taurus en este medio ambiente hostil. Sin embargo, la desventaja de la genética del ganado cebú es que son animales más temperamentales, nerviosos y en su momento agresivos. Debido a la demanda creciente de una mayor eficiencia en los sistemas de producción pecuaria en el trópico se ha incrementado de una manera exponencial el uso de ordeña mecánica y así mismo la aplicación exógena de oxitocina para apoyar la eyección de leche como medida correctiva de un manejo inadecuado durante el ordeño en estas cruzas de ganado cebú.

La práctica de inyectar diariamente oxitocina sintética durante toda la lactación de las vacas ha ido en aumento en los sistemas de doble propósito en las zonas tropicales de México (Villa Godoy et al. 2003). El uso de la oxitocina puede mejorar la eyección de leche sobre todo en aquellos ranchos donde existe un mal manejo en la sala de ordeña e incrementar la leche vendida hasta un $12 \%$ (Nostrand et al. 1991). Incluso si se aplican mayores concentraciones de oxitocina la eyección de 
leche puede incrementarse aún más. Kaskous et al., (2006) reportaron que al inyectar 50 UI de oxitocina a vacas Syrian Shamy se incrementó la producción de leche un $58 \%$.

Aunque la mayoría de los ganaderos tiene la creencia de que las vacas en la sala de ordeña se acostumbran a la inyección diaria y a veces hasta 2 veces al día de oxitocina Garcez et al. (2013) reportaron que estas vacas incrementan las concentraciones séricas de cortisol interpretándose como estrés a la inyección diaria de oxitocina y que nunca se habitúan a este estímulo negativo. El objetivo de este estudio fue determinar el efecto de la aplicación intramuscular de oxitocina para inducir la eyección de leche en indicadores de estrés y temperatura rectal en vacas ordeñadas mecánicamente en el trópico veracruzano

\section{MATERIALES Y METODOS}

El estudio se realizó en un rancho comercial en el municipio de Tihuatlán, Veracruz. Las vacas se manejaron en un sistema de rotación intensiva de 23 hectáreas y 53 divisiones, además se les proporcionó zacate Taiwán picado, ensilaje de naranja, sales minerales y agua fresca a libre acceso.

Después del parto las vacas se asignaron aleatoriamente en 2 grupos: 1) Vacas $(n=56)$ que recibieron una inyección de 10 UI de oxitocina intramuscularmente en la región de la grupa, al ordeñarse y 2) Vacas $(n=40)$ que no reciben la inyección de oxitocina. Las vacas fueron ordeñadas mecánicamente dos veces al día (5:30 am y 2:30 pm). A todas las vacas durante los ordeños se les proporcionó un suplemento alimentico al $18 \%$ de proteína cruda a razón de $1 \mathrm{~kg}$ por 3 litros de leche producida por día. Al entrar al cajón de ordeña las vacas fueron entrampadas de la cabeza. Frente a la trampa las vacas tenían un comedero que les permitió alimentarse durante el procedimiento. Una vez entrampadas las vacas fueron maneadas. Enseguida se procedió a la rutina de limpieza de los pezones y conexión de la unidad de ordeña. Al ordeñarse las vacas se tomaron los siguientes datos: Número de veces que patean. Como lo describe Rounsing et al. (2004) que relaciona el pateo durante la ordeña como señal de estrés. También Wenzel et al. (2003) relacionó la frecuencia de patear en la sala de ordeño con un incremento en la frecuencia cardíaca y las concentraciones de cortisol en leche. En este caso el pateo se utilizó como indicador de estrés en respuesta la inyección de oxitocina durante el ordeño, pisoteo y el número de repeticiones de este evento, si la vaca dejó de comer en respuesta a la inyección, si las vacas vocalizaron, orinaron o defecaron. Al final del ordeño se tomó la temperatura rectal de la vaca utilizando un termómetro digital de rápida medición. Spiers et al. 2001 relacionaron el incremento de temperatura rectal con la frecuencia respiratoria.

Análisis Estadístico. Se utilizó ANOVA y Ji-cuadrada del programa software STATISTICA 7 utilizando tratamiento y número de partos como variables independiente $\mathrm{y}$ las variables dependientes fueron mencionadas en la metodología.

\section{RESULTADOS}

Las vacas inyectadas con oxitocina tuvieron un mayor $(\mathrm{P}=.05)$ porcentaje de animales que pisotearon y patearon (53\%), dejaron de comer $(61 \%)$, movieron la cola $(71.4 \%)$ y se movieron bruscamente $(71.5 \%)$ comparadas con las vacas no inyectadas $(10 \%$ pisotearon, $0 \%$ patearon, $3 \%$ dejaron de comer, $3 \%$ movieron la cola, y $6 \%$ se movieron bruscamente (Cuadro 1). 
Cuadro 1. Indicadores de estrés como respuesta a la aplicación intramuscular de oxitocina para inducir la eyección de leche en vacas lecheras del trópico veracruzano.

\begin{tabular}{|c|c|c|c|c|c|}
\hline Tratamiento & Pisoteo & Pateo & $\begin{array}{c}\text { Dejar de } \\
\text { comer }\end{array}$ & $\begin{array}{c}\text { Mover } \\
\text { cola }\end{array}$ & $\begin{array}{c}\text { Moverse } \\
\text { bruscamente }\end{array}$ \\
\hline $\begin{array}{c}\text { Vacas inyectadas con } \\
\text { oxitocina }\end{array}$ & $53 \%$ & $53 \%$ & $61 \%$ & $71.4 \%$ & $71.5 \%$ \\
\hline $\begin{array}{c}\text { Vacas NO inyectadas } \\
\text { con oxitocina }\end{array}$ & $10 \%$ & $0 \%$ & $3 \%$ & $3 \%$ & $6 \%$ \\
\hline $\mathrm{P}=$ & .05 & .05 & .05 & .05 & .05 \\
\hline
\end{tabular}

pisotearon un mayor $(\mathrm{P}=.05)$ numero de veces (2.62 \pm .36$)$ comparadas con las vacas no inyectadas $(.24 \pm .54)$. También se encontró una interacción $(\mathrm{P}=.05)$ tratamiento $\mathrm{x}$ numero de partos en donde las vacas primíparas inyectadas con oxitocina pisotearon más veces $(4.4 \pm .48)$ que las vacas multíparas inyectadas $(1.1 \pm .54)$; sin embargo todas las vacas inyectadas con oxitocina tanto primíparas como multíparas pisotearon un mayor numero de veces comparadas con las vacas no inyectadas $(.08 \pm$ .73 primíparas y $.40 \pm .80$ multíparas).

Las vacas inyectadas con oxitocina tuvieron una mayor $(\mathrm{P}=.05)$ temperatura rectal $\left(39.13 \pm .08^{\circ} \mathrm{C}\right)$ comparado con las vacas no inyectadas $\left(38.96 \pm .12{ }^{\circ} \mathrm{C}\right)$. También se encontró una interacción $(\mathrm{P}=.05)$ de tratamiento $\mathrm{x}$ numero de partos donde las vacas multíparas inyectadas con oxitocina tuvieron temperaturas rectales mas altas $\left(39.20 \pm .12{ }^{\circ} \mathrm{C}\right)$ comparadas con vacas primíparas inyectadas con oxitocina $\left(39.06 \pm .16^{\circ} \mathrm{C}\right)$ pero ambas tuvieron temperaturas rectales mayores que las vacas no inyectadas con oxitocina (primíparas $38.98 \pm$ $.17^{\circ} \mathrm{C}$ y multíparas $38.94 \pm .18^{\circ} \mathrm{C}$ ).
La inyección intramuscular de oxitocina en la región de la grupa para inducir la eyección de leche en vacas del trópico de Veracruz incrementó todos los indicadores de estrés de estas vacas. Esto es corroborado por Rousing et al. (2004) quienes reportaron que indicadores como pisoteo, pateo, dejar de comer, mover la cola, indicaban estrés y dolor en las vacas y sobre todo se manifestaban de esta manera en respuesta al ser humano.

Wenzel et al. (2003) encontraron una fuerte asociación entre un incremento de pisoteo, ritmo cardiaco y altas concentraciones de cortisol en sangre, sugiriendo que las vacas inyectadas con oxitocina podrían tener un alto grado de estrés.

Las vacas primíparas inyectadas con oxitocina mostraron un incremento mayor en los indicadores de estrés comparadas con las vacas multíparas también inyectadas con oxitocina. Estas reacciones son similares a las reportadas por Jacobs y Siegford (2012) quienes publicaron que las vacas primíparas bajo estrés en la sala de ordeño pisoteaban y 
pateaban más que las vacas adultas bajo las mismas condiciones estresantes.

Jacobs y Siegford (2012) también reportaron que las vacas lecheras ordeñadas en un lugar ajeno al que ellas estaban familiarizadas eran capaces de adaptarse muy rápido y el $60 \%$ de las vacas se adaptaron en 24 horas y en 8 días el $95 \%$ de ellas. Sin embargo en nuestro estudio no importó los días en ordeña y tiempo que fueron inyectadas las vacas, ya que estas siempre reaccionaron negativamente a la inyección intramuscular y rutinaria de oxitocina.

\section{CONCLUSIONES}

Las vacas inyectadas con oxitocina manifestaron elevados indicadores de estrés y temperatura rectal comparados con los de las vacas no inyectadas, sugiriendo que si existe una reacción de las vacas inyectadas intramusculares y rutinariamente con oxitocina y estas no se habitúan a la inyección.

\section{LITERATURA CONSULTADA}

Garcez, N., Domínguez, B., Ángeles, A. A., Torres, H., Alarcón, M. A., Daniel, I. C. y Lammoglia, M. A. 2013. Concentraciones de cortisol y producción láctea en vacas de ordeño inyectadas con oxitocina en el trópico veracruzano. Memorias XVI Congreso Latinoamericano De Buiatría. 249 p.

Jacobs, J. A. y Siegford, J. M. 2012. Lactating dairy cows adapt quickly to being milked by an automatic milking system. J Dairy Sci., 95(3): 1575-84. https://doi.org/10.3168/jds.2011-4710

Kaskous, S. H., Weiss, D. Y., Massri, A. M., Al-Daker, B., Nouh, A. D. y Bruckmaier, R. M. 2006. Oxytocin release and lactation performance in
Syrian Shami cattle milked with and without suckling. J. Dairy Res., 73: 2832.

https://doi.org/10.1017/s0022029905001329

Nostrand, S. D., Galton, D. M., Erb, H. N. y Bauman, D. E. 1991. Effects of daily exogenous oxytocin on lactation milk yield and composition. J. Dairy Sci., 74:2119-2127. https://doi.org/10.3168/jds.s0022-0302(91)78384-7

Rousing, T., Bonde, M., Badsberg, J. H. y Sorensen, J. T. 2004. Stepping and kicking behaviour during milking in relation to response in human-animal interaction test and clinical health in loose housed dairy cows. Livestock Production Science, 88: 1-8. https://doi.org/10.1016/j.livprodsci.2003.12.001

Spiers, D. E., Spain, J. N., Leonard, M. J. y Lucy, M. C. 2001. Effect of cooling strategy on dairy cow performance during heat stress. In R. R. Stowell, R. Bucklin, \& R. W Bottcher (Eds.), Livestock Environment VI: Proceedings of the 6th International Symposium (ASAE Pub. 701P0201. pp. 45-55. https://doi.org/10.13031/2013.7059

Sumano, H. S. y Ocampo, L. 2006. Farmacología Veterinaria. $3^{\mathrm{a}}$ ed. México: McGraw-Hill Interamericana; 2006. pp. 916-919.

Villa-Godoy, A., González-Padilla E. y Ortiz-

Díaz, R. 2003. Oxitocina y somatotropina como método para incrementar la producción en ganado de trópico. En CD-ROM memorias: XVII Congreso Nacional de Buiatría. Villahermosa, Tabasco, México.

Wenzel, C., Scho“nreiter-Fischer, S. y Unshelm, J. 2003. Studies on step-kick behaviour and stress of cows during milking in an automatic milking system. Livestock Prod. Sci. 83: 237-246. https://doi.org/10.1016/s0301-6226(03)00109-x 
Copyright (c) 2014 Miguel Angel Lammog lia Villagomez, Estrella Matinez B., Brablio Aedo Y Loya, Iliana Del Carmen Daniel Renteria y Nora Garcez

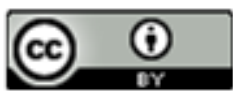

Este texto está protegido por una licencia Creative Commorr 4.0 .

Usted es libre para Compartir —copiar y redistribuir el material en cualquier medio o formato- y Ad aptar el documento —remezclar, transformar y crear a partir del material - para cualquier propósito, inchso para fines comerciales, siempre que cumpla la condición de:

Atribución: Usted debe dar crédito a la obra original de manera adecuada, proporcionar un enlace a la licencia, e indicar si se han realizado cambios. Pvede hacerlo en cualquier forma razonable, pero no de forma tal que sugiera que tiene el apoyo del licenciante o lo recibe por el usoque hace de la obra.

Resumende licencia - Textocompletodklalicenia 